\title{
Adopting an organised and cohesive team-based approach
}

\author{
By Rebecca Ball and Elizabeth Barry, Specialty Dentists in Oral Surgery, King's \\ College Foundation Trust
}

\section{$\mathrm{T}$} he closure of dental practices due to the COVID-19 pandemic has

led to an increase in demand for access to emergency dental care. We wanted to share our experiences of working in an oral surgery department during this period, and the effects this has had on the working environment.

One of the most noticeable and positive things to come out of this pandemic period has been the sense of teamwork and camaraderie within the department. A rota was implemented involving two separate teams of clinicians in order to minimise contact within the team and to aid in enabling social distancing. In addition, the dental nurses also adopted a rota system, with a morning and afternoon team who stayed in separate areas. All team members have shown flexibility and compliance with such measures, including staggering lunches and breaks as needed. This has ensured that social distancing is maintained, whilst keeping the service running smoothly and efficiently.

We begin each day with a team 'huddle', enabling any staff members to provide updates on the working situation and providing opportunity to feed back any thoughts or feelings for the day ahead, as well as any positive comments. This further strengthens the already supportive environment, and ensures all team members are clear on their roles for the day.

Having a lead clinician carry out preoperative checks and provide postoperative instructions to the patients has meant reduced stress and workload for those clinicians carrying out treatment. We have operated in such a way that all team members have felt comfortable and able to rotate between different roles as needed. This has been particularly important when working during the pandemic crisis as the PPE can become uncomfortable after a length of time, which varies from person to person. In addition, the 'buddy system' used in surgeries to ensure PPE is correctly worn has been highly effective and has further bonded the team, particularly between nurses and clinicians.

Morale has been high, and this has been boosted further by positive feedback from our patients. They have been very grateful for treatment received and relief of pain in such a challenging time. By adopting an organised and cohesive team-based approach, the department has been able to successfully serve our local and wider community with emergency dental care. The future is still uncertain, but we have felt privileged to be able to offer much needed emergency dental treatment to our patients during these unprecedented times. \section{tracheostomy service evaluation}

Surgical specialties launch national COVID-19

\author{
The Federation of Surgical Specialty \\ Associations (FSSA) has launched a \\ UK-wide database to evaluate and track \\ the effectiveness of tracheostomies \\ on COVID-19 patients. Support for \\ the Research Data Collection Service \\ (REDCap) is provided by the British \\ Association of Oral and Maxillofacial \\ Surgeons (BAOMS) and ENT UK. \\ The FSSA represents the whole family \\ of surgery and includes the Association of \\ Anaesthetists. It is in the perfect position \\ to promote this important national service \\ evaluation of tracheostomies in patients \\ with COVID-19. \\ The original database was conceived \\ by UCL (University College London) and \\ has now been adopted for roll out across \\ the four countries of the UK. Support
}

for REDCap registration from BAOMS and ENT UK ensures that registration to use REDCap does not delay data collection. The contact for BAOMS is redcap@baoms. org.uk and at ENT UK is covid.trach@ nhs.net. It is expected that there will be enormous demand for registration. There is also an NFORC direct link to use whilst waiting for your REDCap registration to enter data directly: https://trials.nforc.co.uk/ surveys/?s=DL4MWX8TYX.

More information about this project is available on the FSSA website and on the websites of ENT UK and BAOMS.

Kathleen Ferguson, President of the Association of Anaesthetists, said: 'Many patients critically unwell with COVID-19 infection may need a tracheostomy to facilitate their recovery from prolonged

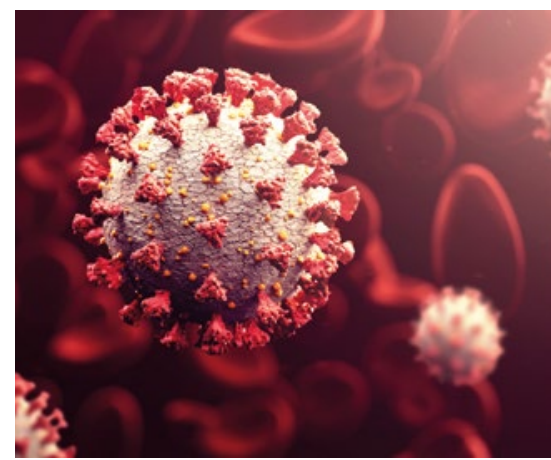

mechanical ventilations. The decision to perform a tracheostomy including the timing and technique is a shared one between the intensive care team and the surgeon. A multi-disciplinary evaluation of this service is essential to add to our understanding of the best care for patients with critical COVID-19. I encourage every unit to sign up to this project.' 